\title{
त्री \\ Estudios sobre ciencia, tecnología y sociedad: en favor del compromiso político
}

\author{
Horacio Correa Lugero
}

\begin{abstract}
电
RESUMEN

El artículo menciona el giro en favor de los enfoques participativos o comprometidos políticamente en el campo CTS, realizando una propuesta para su profundización desde una senda alineada con la teoría crítica de la tecnología. Inspirado en los aportes de Andrew Feenberg y Johan Söderberg, el artículo emprende esta tarea mediante la conjunción de conceptos de la perspectiva de la construcción social de la tecnología con aquellos de una tradición hegeliano-marxista interesada en visiones del sistema capitalista como una totalidad que limita la contingencia en el desarrollo tecnológico. Así, propone el rescate de conceptos como clase, capitalismo y lógica del capital, para la comprensión del desarrollo tecnológico. En el curso de esta tarea, el relativismo constructivista es considerado un problema central para profundizar este giro y se lo reemplaza por una evaluación de los códigos técnicos y del contexto social más amplio que el capitalismo representa. En el trascurso del texto, se expone una reutilización de los conceptos constructivistas con arreglo a los intereses de una posición alineada con la teoría crítica de la tecnología. Así, el concepto "marco tecnológico" se asociará con el de “código técnico", de Feenberg, y con el rescate de la lógica mercantil pregonada por la teoría crítica y Söderberg.
\end{abstract}

Palabras-clave • Giro participativo. Compromiso político. Teoría crítica de la tecnología.

Scot. Marco tecnológico. Código técnico. Lógica mercantil. Lógica del capital. Capitalismo.

\section{INTRODUGGIóN}

La centralidad de las tecnologías resulta evidente en las sociedades contemporáneas. Los estudios sociales de la tecnología se han valido de esta evidencia para poder florecer y desarrollarse sin inconvenientes. De manera similar a cualquier otra disciplina científica, su propio curso se ha alineado a los fenómenos y procesos sociales más amplios en los que cobra vida y se desenvuelve. En un mundo que asume y enfrenta cambios, el campo CTS (ciencia, tecnología y sociedad) vive un giro hacia la generación de propuestas normativas o más participativas, un giro que muestra signos de importancia e interés hacia el compromiso político y en contra de la asepsia política. 
Si bien este giro no se representa en la generalidad y totalidad de los casos, representa un hecho concreto, afirmado y enunciado por diferentes autores en los ámbitos tradicionales de presentación de ideas en el área, a saber, revistas tales como Science, Technology \& Human Values, Social Epistemology o, incluso, Minerva, perteneciente al área filosófica. Esto, vale aclararlo, se ha afirmado fundamentalmente en el ámbito anglosajón. Jasanoff (2003) lo ha calificado de giro participativo, Lynch y Cole (2005) de giro normativo y Söderberg (2010), por su parte, de giro en favor del compromiso político. Más allá de las coincidencias, particularidades y matices de la definición de estos giros, en el presente texto me alineo, en términos básicos, con las características y requerimientos del último autor.

En este sentido, el presente artículo hace mención a ese giro para proponer una perspectiva específica: frente a la posibilidad de elegir entre dos posiciones o tradiciones filosóficas, esto es, frente a una posición relativista y opuesta a conceptos generales, como lo es la constructivista (la tradición de mayor desarrollo en las décadas del ochenta y noventa en el campo CTS) y frente a otra caracterizada por una inclinación hacia términos globales y totalizadoras heredera de la tradición filosófica hegelianomarxista, el presente texto se ha orientado hacia la segunda de las sendas (siguiendo en este sentido el camino emprendido por Söderberg). Por ello, el artículo expone los elementos del constructivismo, básicamente los propios del enfoque de la construcción social de la tecnología, para criticarlos a la luz de las exigencias de la segunda de las sendas. En otras palabras, el presente texto es una apuesta a la profundización del giro mencionado mediante la crítica a una tradición de gran importancia en los estudios sociales de la tecnología y, a su vez, mediante la apuesta a la tradición de la teoría crítica de la tecnología.

La adopción de criterios generales y estructurales orientará el trabajo hacia la crítica de todos los conceptos del enfoque de la construcción social de la tecnología, proponiendo rescatar aquellos susceptibles de ser reorientados hacia la crítica social. Todo el texto, de esta forma, es una defensa de criterios estructurales generales y de conceptos pertenecientes a, y relacionados con, la tradición de la teoría crítica de la tecnología, donde la obra de Andrew Feenberg juega un papel central. Por ello, los conceptos de "clase social”, "código técnico", además de la identificación de la lógica del capital, son propuestos como marco general donde se desenvuelven los demás conceptos. La relación a ellos aparecerá, por lo tanto, como algo fundamental.

De este modo, el presente texto comienza por presentar el giro en favor de los enfoques comprometidos políticamente. Posteriormente, en la sección 2, continúa con una exposición básica y general del enfoque constructivista bajo análisis, señalando sus aspectos críticos y aquellos que presentan mayores inconvenientes para un giro 
afín a las bases filosóficas de la teoría crítica de la tecnología. La sección 3 funciona como síntesis de los elementos a conservar y a rechazar, presentando, adicionalmente, aquellos nuevos elementos a desarrollar.

\section{EL GIRo EN FAVOR DE ENFOQUeS GOMPROMETIDOS POLÍticamenTE}

Durante la década del noventa la corriente constructivista fue de la mayor importancia y desarrollo dentro de los estudios sociales de la ciencia y la tecnología. Su programa específico comienza a tomar forma en la década previa, en los ochenta, para dominar la escena en la siguiente. En su interior, se han desarrollado diferentes vertientes, describiéndose usualmente las siguientes tres líneas fundamentales. En primer lugar, la perspectiva de la construcción social de la tecnología (conocida como Scot por sus siglas en inglés), liderada por Wiebe Bijker y Trevor Pinch. En segundo término, la teoría del actor-red (conocida como ANT por sus siglas en inglés), con el protagonismo de Michel Gallon, Bruno Latour y John Law. Y en tercer lugar, la teoría de los grandes sistemas tecnológicos, con su principal referente en Thomas Hughes.

Más allá de sus divergencias o similitudes (las que, de ser analizadas en profundidad, significarían una extensa labor de clasificación incluso al interior de estas tres grandes líneas mencionadas), el protagonismo constructivista ha venido en descenso desde el inicio del nuevo siglo, llegando a producirse un nuevo escenario que exhibe mayor predisposición para la crítica de la tecnología, ya sea a través de perspectivas feministas, weberianas, institucionalistas, reconstructivistas o afines a la teoría crítica. A la luz de este nuevo ambiente, hace tiempo viene afirmándose que se ha producido un giro normativo en el campo CTS anglosajón (cf. Lynch \& Cole, 2005; Söderberg, 2010). En cierta forma, el cambio de perspectiva también fue entendido en el concepto de giro participativo propuesto por Jasanoff (2003). Una evidencia de esto se encuentra en los llamados en favor "de una postura más activista" destacados en el "discurso presidencial en la reunión de la Society of Social Studies of Science (publicados luego en Bijker, 2003) y en el programa de la reunión de 2002 de la Asociación Europea para el Estudio de la Ciencia y la Tecnología (Easst)" (Lynch \& Cole, 2005, p. 298, nota 2). Acerca del giro normativo, Lynch y Cole (2005) han sostenido que resulta

de difícil reconciliación con las concepciones de la ciencia y de la tecnología construccionistas, reflexivistas y relativistas. Así, por ejemplo, la "simetría” - la ahora clásica idea acerca de que los sociólogos del conocimiento científico deben abstenerse de prejuzgar la verdad o falsedad de creencias particulares antes de 
dar explicaciones de su predominio y distribución - puede difícilmente reconciliarse con un esfuerzo por desarrollar los estudios CTS como una base para realizar intervenciones normativas en debates públicos sobre riesgo, salud, seguridad, efectividad, o eficiencia (Lynch \& Cole, 2005, p. 270).

Este giro, como lo señala Söderberg (2010), podría considerarse más concretamente un giro hacia unos estudios sociales de la ciencia y la tecnología con mayor compromiso político, donde la clásica distinción caracterizada por Steve Fuller (1993) entre una "alta iglesia" de estudios CTS y una "baja iglesia" estaría por desaparecer o converger en un "programa comprometido" (cf. Sismondo, 2008, p. 21; Söderberg, 2010, p. 24,2).

Estos cambios también se han manifestado en el ámbito constructivista argentino, donde los principales referentes han tenido una reorientación hacia el estudio de las llamadas tecnologías sociales. Esta idea puede sostenerse a través de la lectura de la definición que de tecnología social da uno de ellos:

Es posible definir Tecnología Social como una forma de diseñar, desarrollar, implementar y gestionar tecnología orientada a resolver problemas sociales y ambientales, generando dinámicas sociales y económicas de inclusión social y de desarrollo sustentable (Thomas, 2009, p. 2; énfasis mío).

Esta es la senda que ha tomado el constructivismo argentino en favor de la intervención en el desarrollo de tecnologías intencionadamente orientadas a la resolución de problemas sociales y ambientales específicos. Sin embargo, es necesario aclarar que de ningún modo representa una negación de los principios previos, sino que, por el contrario, este cambio significa una profundización de la posición constructivista, caracterizada como un abordaje totalmente coherente con perspectivas políticas de análisis. Una frase lo deja totalmente claro:

El constructivismo deja de ser una cuestión exclusivamente teórica metodológica limitada al ámbito académico y pasa a constituirse en una forma de profundización de las relaciones democráticas. La apertura de cajas negras - operacionalizada por los abordajes constructivistas - es tanto una herramienta heurísitica como una estrategia política (Thomas, Fressoli \& Lalouf, 2008, p. 74).

Esta característica del constructivismo en Argentina parece no seguir las líneas que se observan en la literatura anglosajona, donde algunos clásicos protagonistas (cf. Latour, 2004; Law, 2009) "han comenzado a tener segundos pensamientos sobre sus 
ideas tempranas sobre ciencia y política" (Söderberg, 2010, p. 24,2), lo que ha significado la asunción de la necesidad de romper con sus propias raíces en favor de un compromiso político. En el plano argentino, de este modo, parecieran no ofrecer inconvenientes o contradicciones ni el relativismo característico de esta corriente, ni el principio de simetría, dos elementos que sí parecieran generar ciertos problemas en el ámbito donde estos estudios se originaron.

Ahora bien, lo que interesa de todo esto no son los problemas internos del giro, ni tampoco hacer una revisión de cambios y permanencias en la tradición constructivista frente a los nuevos tiempos, sino, por el contrario - y además de destacar las potencialidades de un entorno que se muestra a favor de contribuciones comprometidas políticamente -, interesa en el presente texto realizar un análisis de las vías asumidas por el giro en favor del compromiso político. En otras palabras, si ha habido un giro o si, al menos, este giro parece tomar fuerza, interesa saber: ¿cuáles son sus aspectos más importantes para la consolidación de una crítica radical de la tecnología? Y, asimismo, ¿cuáles son los aspectos que conviene olvidar de la tradición en declive? En definitiva, siendo que se está produciendo un giro, y además, que este giro parece eliminar la vieja oposición entre "alta iglesia" y "baja iglesia” de estudios CTS, interesa analizar cuáles son los aspectos que mejor pueden contribuir al establecimiento y afianzamiento de una senda de estudios en favor de análisis críticos y radicalizados de las tecnologías y la sociedad. Para comenzar a tratar esta problemática, pasemos a continuación a una rápida revisión sobre los aspectos básicos de la visión del enfoque Scot.

\section{El enfoque Scot y sus Problemas}

\section{2.l Aspectos BÁsicos}

Dentro de los enfoques constructivistas el de la construcción social de la tecnología (Scot) ha desarrollado una serie de conceptos que permiten dar cuenta de los procesos de construcción de las tecnologías en nuestras sociedades. ${ }^{\mathbf{1}}$ Todos estos conceptos se

\footnotetext{
1 He seleccionado aquí primordialmente el enfoque Scot, debido a que es éste el que posee algunos elementos mínimos que deseo resaltar en la perspectiva que aquí propongo. Siendo una de las tradiciones más importantes de las últimas décadas creo que es fundamental reconocer aportes indispensables de esta tradición. La crítica aquí expuesta, por lo tanto, debe ser entendida en este sentido y, adicionalmente, como un intento de criticar los aspectos que pueden atentar contra el giro en favor de las posturas comprometidas políticamente en los estudios de la tecnología. De las otras dos perspectivas mencionas, es decir, de la de los grandes sistemas tecnológicos y de la TAR, sólo me remito brevemente a la primera en el punto 2.2.2. Una visión de "actantes", en un ambiente de "simetría radicalizada", como sucede en la TAR, no deja márgenes para la reapropiación crítica en los sentidos del presente texto, algo que, aunque mínimamente, permite el enfoque Scot.
} 
desarrollan en un marco relativista y de pretensión simétrica en el sentido del principio de simetríadel Programa fuerte de la sociología del conocimiento científico. ${ }^{2}$

Uno de los conceptos principales es el de "grupo social relevante" y representa a cada uno de los sujetos o entidades que forman parte de las relaciones de poder que da forma a una tecnología específica. Cada grupo social relevante será agrupado de acuerdo a los significados que le otorgue al artefacto o tecnología en construcción. Bijker ha señalado que cada grupo social relevante no sólo ve aspectos diferentes de un artefacto, sino que, más precisamente, "construye" un artefacto diferente (Bijker, 1995, p. 77). El concepto de grupo social relevante está en estrecha relación con otro desarrollado por el enfoque Scot, el de "flexibilidad interpretativa”. Es justamente el momento de significaciones diferentes de un artefacto en construcción, de enfrentamiento entre las diferentes visiones y construcciones de éste, el que recibe el nombre de "flexibilidad interpretativa". Es durante el desarrollo de la flexibilidad interpretativa cuando encontramos tantos significados del artefacto cuantos grupos sociales relevantes existan. Por su parte, el momento en que esta flexibilidad interpretativa decae recibe el nombre de "clausura". La clausura indica el surgimiento del consenso en los significados atribuidos y, por ello, el momento en que la flexibilidad interpretativa deja de existir. Los artefactos, una vez que han llegado a la clausura, son difícilmente modificables. Sin embargo, como las posibilidades aún pueden llegar a existir, la perspectiva Scot ha propuesto el concepto de "estabilización” o "grados de estabilización”. Específicamente, la estabilización representa el período en que el significado del artefacto se torna estable hacia el interior de un grupo social relevante. Como existen diferentes grados y momentos de homogeneización de los significados atribuidos a un artefacto en construcción, el enfoque Scot presenta el concepto de "grados de estabilización”. Este concepto permite, además, introducir el elemento de dinamismo y de cambio propio de las tecnologías.

Ante las críticas a las primeras versiones de la propuesta por su falta de remisión a cuestiones estructurales que limitaran los significados otorgados, Bijker (1995) propuso el concepto de "marco tecnológico". Un marco tecnológico es aquello que "provee los objetivos, las ideas y las herramientas necesitadas para la acción” (Bijker, 1995, p. 192), el que guía el pensamiento y la interacción, ofrece los problemas centrales y las estrategias para resolverlos (cf. Bijker, 1995, p. 125). Además,

\footnotetext{
2 Este principio fue presentado originalmente por Bloor $\left(197^{3}\right.$, 1991) para el campo de la sociología del conocimiento científico. Luego fue adoptado por la sociología de la tecnología. Según Bloor, los sociólogos del conocimiento científico debían ser imparciales con respecto a la verdad o falsedad de las creencias. Es en este sentido que él establece que la verdad y la falsedad deberían ser analizadas igualmente, en forma simétrica, sin privilegiar una sobre la otra. "Pinch y Bijker (1984) extendieron este principio al análisis de la tecnología, argumentando que el funcionamiento o no funcionamiento de las máquinas sea analizado simétricamente” (Bijker, 1993, p. 119).
} 
Dentro de un marco tecnológico ya no es todo posible (el aspecto de estructura y tradición), pero las posibilidades remanentes están relativamente clara y fácilmente disponibles para todos los miembros del grupo social relevante (el aspecto del actor y de la innovación) (Bijker, 1995, p. 192).

El marco tecnológico es el que permite la acción, el desarrollo de tecnologías con sentidos específicos, pero es el que, a la vez, constriñe la libertad de los miembros del grupo social relevante. Es el que provee, por lo tanto, las finalidades e intenciones a los grupos sociales relevantes. Siendo estos los aspectos más básicos de esta perspectiva, propongo pasar a su crítica.

\subsection{Crítica al enfoque Scot y propuestas}

\subsubsection{Grupos SOGIALES RELEVANTES: LA NEGESIDAD DE GUESTIONES ESTRUGTURALES}

Realizado este repaso veloz, es pertinente pasar a una crítica de estos elementos para poder, incluso con ello, profundizar sobre algunas implicaciones del herramental teórico de esta perspectiva.

Para comenzar, pueden mencionarse los problemas del concepto "grupo social relevante". El primero de ellos radica en la indiferenciación al interior de la sociedad que concibe. Con este concepto,

Implícitamente, [el enfoque] Scot asume que todos los grupos son iguales y que todos los grupos sociales relevantes están presentes en el proceso de diseño. Lo cual falla en dar cuenta adecuadamente de las asimetrías de poder entre grupos (Klein \& Kleinman, 2002, p. 29).

Este problema parte del hecho de considerar a la sociedad como conformada por acciones singulares o particulares, de individuos o grupos que pueden "actuar" en todo momento sin mayores restricciones que las impuestas por los conflictos ocasionados en la flexibilidad interpretativa misma, o por los marcos tecnológicos. La consideración de la sociedad o la cultura como elementos incidiendo en la conformación de los grupos sociales relevantes conduciría al sostenimiento de un determinismo social, una aberración para el esquema constructivista. De ahí, los problemas de poder identificar diferenciaciones estructurales entre grupos sociales relevantes. De esto se sigue la conexión entre grupos sociales relevantes y la falta de consideraciones estructurales amplias.

La identificación estructural, consecuentemente, permite comprender e incluir diferencias entre grupos. Realizar una identificación de tipo estructural de la perte- 
nencia de esos grupos sociales permite dar cuenta de incidencias externas más allá de lo que sucede internamente en los grupos o en la dinámica de la relación entre grupos durante la "flexibilidad interpretativa". De este modo, la inclusión de la estructura permite evaluar la incidencia del "entorno", en otras palabras, los modos de inserción de lo particular en lo universal. Un ejemplo de ello puede verse en Rosen (1993), quien expone el modo en que el "contexto posmoderno de la economía post-fordista (...) explica el diseño y continuo rediseño de las mountain bikes" (Klein \& Kleinman, 2002, p. 31). La incidencia de los sistemas de "producción flexibles que emergieron a mediados de los setenta" resultan fundamentales para comprender ese constante rediseño (Klein \& Kleinman, 2002, p. 31).

Grupos sociales relevantes por sí mismos no bastan para dar cuenta de situaciones como la descripta por Rosen, sus relaciones micropolíticas no son suficientes para comprender la dinámica del cambio tecnológico. Tampoco es suficiente la incorporación de un marco tecnológico, puesto que, si bien es importante para dar cuenta de constricciones a la acción y a los significados dados a las tecnologías, no fue pensado para dar cuenta de incidencias surgidas de un "afuera" más extenso, como lo es la lógica del capital y mercantil en el capitalismo. Viendo el ejemplo de las mountain bikes desde una postura inclusiva de la lógica del capital, la producción flexible bien puede remitirse a modificaciones estructurales en la acumulación de capital. En ese sentido, las modificaciones estructurales de la lógica del capital y la consecuente modificación del modo de acumulación constriñen y orientan posibles modos de producir las tecnologías. En ese abanico de posibilidades es que se desarrollan unas opciones, mientras se inhiben otras. Esto no significa que las acciones estén pre-determinadas, sino que se producen en una suerte de contingencia limitada por una estructura específica.

En este sentido, los grupos sociales relevantes, desde una posición de orientación marxista, tendrían que ser entendidos como representantes de clases sociales o de fracciones de clases, cuyas especificidades se siguen del funcionamiento particular de la lógica del capital y de la acumulación en períodos específicos del capitalismo, como los denomina Marx en sus textos de análisis de las revoluciones francesas (cf. Marx, 2003). Aquí, una primera aclaración es necesaria: esas clases se caracterizan por la inexistencia de posiciones fijas y predeterminadas, es decir, no hay en la categoría de clase posibilidad de pertenencia "rígida, desde siempre y para siempre" (Grüner, 2008, p. 33-4). Adicionalmente, la categoría de clase a la que aquí adhiero reconoce sin problemas la existencia de identidades diversas y cada vez más fragmentarias en nuestras sociedades contemporáneas, sin necesidad de poner en jaque una visión clasista de la sociedad (cf. Grüner, 2008). Esta concepción clasista, en consecuencia, no elimina el hecho de que los sujetos están atravesados por una infinidad de demandas 
de identidad que toman como propias, base sobre la cual constituyen su propia identidad. Ante la duda de la aplicabilidad de la categoría de clase, la siguiente pregunta siempre es pertinente, ¿quién puede negar la existencia de la propiedad privada de los medios de producción, fuente básica de la división clasista de la sociedad? En la respuesta a esa pregunta radica la fuerza de esta categoría.

La defensa de la utilización del concepto de "clase" fue expresada en la filosofía de la tecnología por Feenberg, quien, en Questioning technology, ha expresado más claramente:

El rechazo frecuente de conceptos macro-sociológicos tales como clase y cultura arma aún más la investigación en contra de la política tornando casi imposible introducir los factores de extensión y amplitud social que dan forma a la tecnología a espaldas de los actores (Feenberg, 1999, p. 11).

La utilización conceptual de las clases sociales que aquí propongo contempla dos cuestiones: por un lado, las representaciones de identidad de los miembros de las clases y, por el otro, las posiciones efectivas de los sujetos en las clases. De este modo, la construcción de tecnologías, en términos generales, involucra mixturas de estos dos elementos. Por ejemplo, una identificación con los valores hackers (auto-identificación como hackers) puede conducir a la construcción de alternativas a las tecnologías existentes, pero, a su vez, la posición de clase puede condicionar vías posibles para realizar esa alternativa.

El caso propuesto por Söderberg (2010) sobre el desarrollo de dos proyectos alternativos de redes libres inalámbricas en la República Checa expone estas cuestiones de fondo. Las identificaciones con valores libres, además de los altos costos de los servicios de Internet, condujeron a pensar la posibilidad de desarrollar una red wireless descentralizada mediante la utilización de un espectro de luz visible como medio de transmisión de los datos. Los desarrollos específicos de los dos proyectos en disputa que allí se exponen se encuentran con determinaciones del mercado en el diseño de las tecnologías y con la imposición de unas alternativas de materiales a utilizar sobre otras. En este sentido, el cambio en la masividad de uno de los dos modelos de desarrollo de la red wireless implicó establecer modificaciones de diseño específicas para abaratar costos que, adicionalmente, impusieron transformaciones en los modos de construcción y expansión de la red. Esto propició un ambiente - lo que podría denominarse marco tecnológico - afín a la incorporación de personas que no contribuían como colaboradores, sino sólo como consumidores, cambiando, con ello, la impronta de la red, donde inicialmente todos eran, a la vez que usuarios, colaboradores. 
Adicionalmente, la clase social de pertenencia del líder de uno de los dos proyectos que el texto de Söderberg (2010) presenta (es decir, su posición efectiva en la clase haciendo abstracción de sus auto-identificaciones) lo lleva a encarar estrategias específicas: de su posicionamiento social como asalariado, se siguen estrategias bien claras para poder dejar su trabajo y dedicarle tiempo completo al desarrollo de la red. ${ }^{3}$ Esto demuestra el modo en que su posición efectiva de clase constriñe sus posibilidades concretas de acción, en otras palabras, el modo en que la estructura limita la plena existencia de la contingencia.

\subsubsection{Unos SUJeTos OLVIDADos:}

LOS SUJETOS HETERÓNOMOS Y SU PARTIGIPAGIÓN LIMITADA AL MERCADO

En otro texto, Feenberg (2005) plantea una distinción pensada para dar cuenta de las diferencias de posición existentes en la producción de tecnologías: por un lado, los gerenciadores y, por el otro, los gerenciados. Así, "la tecnología puede ser y es configurada de un modo tal que reproduce el dominio de pocos [los gerenciadores] sobre muchos [los gerenciados]" (Feenberg, 2005, p. 111). Esta visión da lugar a plantear no sólo la existencia de lo que podríamos llamar sujetos heteronomizados, sino que también permite dar cuenta de relaciones de poder no consideradas dentro del esquema desarrollado por el enfoque Scot. Veamos esa idea de otro modo.

El abordaje Scot ha sostenido la capacidad de su visión teórica para demostrar el modo en que se construyen políticamente los artefactos, en este sentido, permite, según ellos en defensa de la utilidad política de su desarrollo conceptual, mostrar que las cosas no son indefectiblemente como se muestran, sino que pueden modificarse. El otro lado de esta afirmación es el reconocimiento de que verdaderamente los sujetos no participan en la creación o construcción de tecnologías, por lo que se encuentran efectivamente heteronomizados frente a objetos que se presentan como autónomos. Esta idea no está representada, en términos generales, por ningún concepto del herramental teórico del enfoque Scot, centrándose por el contrario en cuestiones micro-sociales - en palabras de Feenberg (2005), "abundancia empírica" - y omitiendo la existencia de otra esfera de manifestación del poder, el lado de aquellos que reciben lo construido, no pudiendo reconocer la pasividad de quienes reciben (compran o desean comprar) las tecnologías. ${ }^{4}$ Es decir, omitiendo la consideración del mercado.

3 La presentación de Söderberg es muy clara y llena de detalles, la extensión del presente texto no permite su plena exposición más que en los lineamientos generales y, por ello, más vagos en detalle.

4 Digo "en términos generales", porque Bijker sí ha propuesto un concepto en respuesta a críticas. Pero como dicho concepto no es más que marginal en el esquema general, he decido expresar la idea en estos términos. Ver la nota al pié 9 . 
En esta línea, podría sostener que en el momento de la flexibilidad interpretativa no intervienen las fracciones de clase gerenciadas. Una parte de ellas son, antes bien, las que luego construirán esas tecnologías pero siguiendo los lineamientos demandados por los propietarios de esos objetos técnicos, 5 fundamentalmente, aquellos alineados de parte de alguna de las fracciones de clase que, en términos de Feenberg (2005), son los dominadores o los gerenciadores.

A mi entender, sin embargo, la participación de estos sujetos gerenciados, para utilizar ese término, o de los sujetos heterónomos, se da en un nivel de participación más sublimatorio, esto es, por medio del mercado (percibido por estrategias de marketing). De acuerdo con Zizek, “cada universalidad hegemónica tiene que incorporar por lo menos dos contenidos particulares, el contenido popular auténtico, como así también su distorsión creada por las relaciones de dominación y explotación" (Zizek, 1997, p. 29). Esta idea expresa el modo en que la construcción de hegemonía toma en cuenta las ideas creadas por las masas pero con una distorsión, aquella que permite sostener las relaciones de dominación y explotación: es decir, el capitalismo. Zizek comenta la reversión hecha por Balibar (1997) de la fórmula de Marx para reforzar su argumento, señalando que "las ideas dominantes, precisamente, no son directamente las ideas de quienes gobiernan" (Zizek, 1997, p. 3o).

Transponiendo esas visiones al campo de los estudios sociales de la tecnología, puede consignarse que las ideas de los creadores o constructores provienen de los dominados o fracciones de clase descriptas aquí como heterónomas pero siempre creando una distorsión, aquella que permite sostener las relaciones de dominación y explotación, en definitiva, la lógica del capital funcionando. Esta idea de distorsión puede resultar más que controversial, sin embargo, considero que lo destacable de este argumento de Zizek es que ante cualquier voz contraria a los intereses del sistema capitalista (esto no significa que todas las voces que surjan se le opongan), habrá una reacción tratando de incorporar en esas ideas las condiciones necesarias para sostener las relaciones de dominación y explotación, en definitiva, la continuidad de la valorización del capital.

Por ejemplo, la idea de desarrollar una vida cada vez más "libre" y confortable proviene de los mismos gerenciados, de los sujetos heterónomos. Estas demandas fueron suplidas, en el caso específico de la tecnología, por las grandes empresas que las

5 Su participación es considerada en términos de eficiencia. Se los organiza de tal forma que garanticen una mayor producción. Es sabido que se los observó históricamente con la intención de estudiar sus movimientos y así organizar de forma más eficiente la producción. De esta manera, su participación es indirecta y referida a las contribuciones que de su trabajo surgen para incrementar esta productividad, la que, a su vez, posibilita mejorar en términos relativos la producción con respecto a competidores. Aunque cabe señalar que existe otra modalidad de participación indirecta de los gerenciadores, como muestro más adelante en este apartado remitiendo a Zizek. 
desarrollaban, creando cada vez más dispositivos que le dieron a las personas esa "libertad" y mayores estándares de vida. Pero acompañado a ello vino el disfrute de la propia individualidad, lo que sin lugar a dudas permitió sostener y reforzar poderosamente las relaciones de dominación y explotación, haciéndolas aún más invisibles y sublimes. Celulares con todo lo que uno puede necesitar, consolas de video juegos, entre cientos de tecnologías, nos permiten sumirnos en nuestra individualidad olvidándonos de nuestra existencia como sujetos colectivos, permitiendo, con ello, reproducir las relaciones de dominación sin mayores inconvenientes, es más, viendo a aquellos que sí luchan contra esas relaciones como sujetos que atentan contra nuestra propia "libertad". En este sentido puede hablarse, incluso, de la contribución de la tecnología, si no al fin de lo político, al menos a la reducción de su existencia a algo poco menos que marginal. Pero, nuevamente, las relaciones de clase y la cultura en un momento determinado, junto con las características de la lógica del capital (y los requerimientos del mercado), funcionan como ámbito que constriñe las posibilidades de diseño y construcción de tecnologías. De ese modo, un situación de crisis económica puede orientar una reapropiación de esas tecnologías poniéndolas al servicio de intereses emergentes. Así, la crisis capitalista mundial actual debe ser vista como contextual en el modo en que las actuales redes sociales de la web sirvieron para coordinar acciones en las marchas y movilizaciones espontáneas. A partir de esto, uno estudio del surgimiento del Movimiento 15-M español debería tener en cuenta, por ejemplo, además de las transformaciones estructurales del sistema capitalista que lo llevaron a la crisis y su manifestación en España, los modos en que las plataformas web fueron reapropiadas para ponerlas al servicio de esa lucha política.

Una crítica de la tecnología, en este sentido, tendría que poder dar cuenta de los contenidos particulares de la tecnología y, asimismo, del conjunto de los sujetos heterónomos. Esto es, por un lado, dar cuenta de los valores incorporados a las tecnologías (ver la conexión de esto con el concepto de Feenberg, código técnico, en el apartado 3 del presente artículo) y, por el otro, dar cuenta de la composición de identidad y del posicionamiento social de los sujetos heterónomos.

\subsubsection{El PRoblema DE LA AUTONOMÍA DE LA TEGNOLOGÍA}

Y DE LA INDIFERENGIAGIÓN ABSOLUTA ENTRE TEGNOLOGÍA Y SOGIEDAD

La perspectiva Scot, en su avanzada en contra del determinismo tecnológico, evita una cuestión bien destacada por otro autor constructivista, Thomas Hughes, quien sostuvo la posibilidad de que los sistemas tecnológicos, al madurar, tuvieran incidencias sobre la sociedad, sus grupos, individuos, e incluso, sobre otros sistemas tecnológicos. El concepto para describir esta situación es el de "impulso tecnológico" (Hughes, 
1987). ${ }^{6}$ De esta forma, los sistemas tecnológicos, al adquirir gran impulso tecnológico, tienden a ejercer un determinismo suave sobre otros sistemas o grupos sociales. ${ }^{7}$

Que Hughes (2008) hable de "gran" impulso da cuenta de la existencia de graduaciones o niveles de impulso tecnológico. Así, Hughes afirma que un "alto nivel de momentum (impulso) a menudo produce que quienes lo observan asuman que un sistema tecnológico ha devenido autónomo" (p. 139). Y cierra diciendo, "los sistemas maduros poseen una cualidad que es análoga, por consiguiente, al movimiento inercial" (Hughes, 2008, p. 139). Es decir, la madurez de un sistema tecnológico o su alto nivel de impulso son los que contribuyen a generar una idea de autonomía en el desarrollo tecnológico. Esta autonomía no es aprehendida por el enfoque Scot, debido al rechazo exacerbado de todo tipo de posibles incidencias sobre la sociedad, es decir, de cualquier tipo de determinismo, por más suave y limitado que éste sea. Igualmente, esta falta también es expresión, entiendo, de la afirmación teórica sobre la imposibilidad de separar entre tecnología y sociedad y, en consecuencia, de la falta de consideración de criterios estructurales.

Así, a pesar de la importante contribución que implica la crítica al determinismo en sus formas extremas (incluyendo a aquellas propias del marxismo vulgar), el enfoque Scot deja sin mención - a causa de ello - algo fundamental para avanzar en la crítica de la tecnología en nuestra época, esto es, que las tecnologías, una vez estabilizadas y clausuradas, logran adquirir un impulso tal que se presentan como autónomas ante todos los sujetos. De este modo, producen la heteronomía de quienes las adoptan, de quienes las adquieren, de quienes las incorporan en su vida cotidiana. Con esto quiero decir que, si bien existen grupos participando en la producción o construcción de las tecnologías, éstos son sólo una porción menor de la población, surgiendo así la producción de heteronomía del resto de las personas que no participan en ese proceso (reforzando con esto lo dicho en el punto 2.2.1 sobre sujetos heterónomos).

Esta omisión o no consideración de aquellos grupos o sujetos que sólo son receptores de las tecnologías se asienta, asimismo, sobre los problemas que acarrea la indistinción absoluta entre tecnología y sociedad. Es en la base de esta escisión que es posible distinguir y apreciar la existencia de estos sujetos heterónomos y de la autonomización de la tecnología. De esto modo, "si no podemos distinguir analíticamente

6 En inglés, "technological momentum". Existen traducciones que conservan el término "momentum"; yo he preferido utilizar el término traducido porque me parece que brinda mayor claridad a personas que no manejan el idioma inglés.

7 Los sistemas son torneados por el "constructor de sistemas", una forma de sostener una doble agencia entre sociedad y tecnología y, por demás, un concepto problemático, siendo que detrás del esquema teórico de Hughes se encuentra la idea del tejido sin costuras (seamless web), concepto ideado para dar cuenta de la indiferenciación entre tecnología, sociedad y naturaleza. 
entre contexto y contenido (tecnología), es entonces imposible, incluso en un modo tentativo, comprender cómo el mundo social moldea a (el significado de) los artefactos" (Klein \& Kleinman, 2002, p. 36).

Por lo tanto, si bien puede ser criticada su existencia a través de los estudios micro-sociales iniciados hace décadas por los autores constructivistas, la autonomía de la tecnología cobra vida y sentido en los desarrollos tecnológicos que ya se encuentran asentados en la sociedad. Esto es, en las visiones de tipo estructural. De este modo, la distinción entre tecnología y sociedad también cobra sentido en un nivel macro-social.

\subsubsection{El PRINGIPIO RELATIVISTA COMO SUBSIDIARIO DE LAS NEGESIDADES DEL CAPITAL}

El relativismo del constructivismo también presenta sus inconvenientes, más que nada, para una posición comprometida políticamente. La sociología constructivista critica un discurso científico caracterizado por la impronta objetivista, sin embargo, en su principio de simetría reproduce el mismo discurso "objetivista" criticado en la ciencia. En otras palabras, el relativismo propone dar visiones más objetivas de la construcción de una tecnología o de una teoría científica y en eso reproduce el discurso científico tradicional. Por otro lado, si bien considera un poder semiótico y una micropolítica (cf. Bijker, 1995; Thomas, 2008), no lo hace más que para ver relaciones de poder que construyen "marcos tecnológicos" y se coloca en función de su simetría objetiva científica.

El relativismo propio de las perspectivas constructivistas implica plantear que todas las opciones en el momento de la flexibilidad interpretativa deben considerarse con iguales posibilidades de imponerse y el analista no puede hacer ningún juicio de valor sobre lo que observa. En este sentido, es imposible discutir cuál de los sentidos es mejor, ya sea en términos políticos, económicos o de cualquier otro tipo. De esta forma se hace presente la objetividad, pretendiendo hablar en nombre de ella, defendiendo un modelo racionalista de ciencia en forma implícita, siendo, en este sentido, totalmente subsidiario de las necesidades propias del sistema capitalista, en el sentido que menciono a continuación.

El constructivismo ha construido su perspectiva en forma consecuente con el tiempo en que vivimos. En un mundo acostumbrado a escuchar sobre la muerte de los "meta-relatos", de las grandes construcciones teóricas, ellos construyen su visión en línea con ese nuevo "paradigma". Reconocer que esa nueva forma de razonar es fruto de y directamente vinculable con las transformaciones sociales vividas en el mundo a partir de los setentas es una forma de entender el por qué de semejante posicionamiento ideológico. 
A partir de los setentas se produce una fuerte regresión del poder de las organizaciones de trabajadores, golpes y gobiernos militares atacan directamente sus construcciones y dan pasos enormes en el desbaratamiento de la unidad de los trabajadores. ${ }^{\mathbf{8}}$

Todo esto tuvo notables repercusiones en la notoriedad de las ideologías de orientación marxista, el horizonte se mostró libre de peligros inminentes, todo se libró, ya en los noventas, a la fuerza del mercado. Claramente esto tuvo sus ecos en las teorizaciones y visiones del mundo. El constructivismo debe entenderse como hijo de esas transformaciones, como hijo de un mundo que no ofrece perspectivas instituyentes, como diría Castoriadis (2012), sino manejos dentro del mundo de lo instituido. Con el cambio de siglo y, más aún, con el comienzo de la presente década, los vientos de cambio soplan nuevamente, pero a favor, entre otras cosas, de la constitución de una teoría "crítica" dentro del estudio de la tecnología, a favor del desarrollo de unos estudios de la tecnología comprometidos políticamente.

Esta idea es consecuente con una perspectiva que concibe a la teoría y a su desarrollo como limitados o condicionados por las especificidades de un tiempo histórico particular.

\section{A modo de síntesis: ELEMENTOS Gentrales A PRESERVAR EN UN ENFOQUE CTS GOMPROMETIDO POLÍTICAMENTE Y NUEVOS ASPECTOS A AÑADIR}

En primer lugar es necesario destacar aquellos elementos del enfoque Scot útiles para el desarrollo de unos estudios CTS comprometidos políticamente, para alimentar, con ello, el giro ocurrido en este campo de estudio. Como cada elemento que puede preservarse exige una modificación para su adaptación a este giro comprometido, se presentan conjuntamente los conceptos a presentar y las modificaciones exigidas junto con sus implicaciones generales. Antes de ello, recuerdo brevemente que en esta crítica y en mi asunción teórica están en juego posiciones filosóficas específicas.

Es importante recordar una distinción central y general. Las teorías y perspectivas teóricas en los estudios de la tecnología pueden agruparse en dos grandes grupos, aquellas a favor de la utilización de conceptos totalizantes, y aquellas en contra. Cada uno de estos dos grandes grupos se inscriben en tradiciones filosóficas opuestas:

8 Es sabido también el golpe enorme que significó la caída de la URSS. Sin ir muy lejos, en la propia Argentina ese hecho implicó la inmediata fragmentación de los partidos de izquierda que se orientaban a la búsqueda del socialismo. 
[Por un lado, la] sospecha en contra de afirmaciones de verdad general constituye una parte integral de la corriente intelectual a la que muchas escuelas constructivistas del campo STS [CTS] pertenecen. En contraste, la inclinación en la teoría crítica a utilizar conceptos “totalizantes” puede remontarse hasta Hegel y Marx. La importancia de este debate sobre puntos más bien abstractos y filosóficos de diferencia se torna claro cuando se lo relaciona con análisis de la economía. Una comprensión teórica del intercambio de mercancías como una relación que impregna la totalidad del capitalismo es un punto de inicio de un análisis de la ciencia y la tecnología conducido en la tradición de la crítica teórica (Söderberg, 2010, p. 24,0).

En este sentido, el presente artículo se posiciona claramente en la segunda de las sendas. Por ello, la remisión a la totalidad (como estructura capitalista, donde la lógica del capital juega un papel central) es algo que se ha destacado en la crítica de los conceptos constructivistas.

Consecuentemente, una de las intenciones del artículo ha radicado en la incorporación de elementos estructurales. Esto no significa que las acciones estén pre-determinadas, sino que se encuentran en una suerte de contingencia limitada por una estructura específica. Con esto intento destacar los factores particulares que no pueden apreciarse más que con su investigación explícita y específica, sin dejar, sin embargo, de remitirlos a situaciones contextuales más amplias de mayor envergadura, incluso, que los marcos tecnológicos propios del enfoque Scot. Esas situaciones contextuales más amplias, o contexto más general, han sido identificadas por Feenberg como el "contexto social más amplio". Aquí, ese contexto social más amplio no es otro que el capitalismo y sus reglas específicas de funcionamiento. Replanteado el posicionamiento filosófico y teórico general, pasemos ahora al detalle de las críticas y propuestas.

Lo primero a destacar reside en los análisis micro-sociales que dan cuenta del proceso de construcción de las tecnologías. La apertura de la “caja negra” de la tecnología es central e indispensable para comenzar a pensar en la posibilidad de cambiar el orden social tecnológico que nos rodea. En este sentido, la flexibilidad interpretativa puede utilizarse siempre y cuando dé cuenta de las procedencias sociales y culturales de los significados en disputa. Los conceptos del enfoque Scot podrán utilizarse siempre y cuando se los colonice con posiciones estructurales que consideren la lógica del capital y el mercado como elementos centrales estructurando los significados otorgados a las tecnologías, los marcos tecnológicos y, por lo tanto, la conformación de los grupos sociales relevantes. Ahora bien, ¿qué representa esta estructura que se menciona?

Estructura se utiliza aquí en el sentido más amplio, esto es, en la consideración del capitalismo y de sus principios rectores. El capital como valor en proceso de valo- 
rización. La estructura no implica bajo ningún concepto, como acabo de señalar, determinaciones lineales absolutas que no dejan posibilidad a la contingencia, muy por el contrario, la contingencia existe, pero no en forma plena, sino con las limitaciones propias de la estructura capitalista y de sus valores implícitos.

Profundamente ligado con esto último, surge el mercado como otro elemento central. De ser completamente ignorado en los estudios de la tecnología, es factible ahora plantear su apreciación como fundamental para el análisis. El lado del mercado es central en la conformación y diseño de las tecnologías, tal cual ya lo he sostenido.

Por otro lado, es necesario reconocer la importancia del concepto "grupo social relevante" para dar cuenta de los grupos que participan en el diseño y construcción de las tecnologías, sin embargo, he propuesto incluir diferenciaciones entre grupos bajo distinciones de clases y de identificaciones de identidad. Ambos aspectos combinados pueden dar cuenta de las posiciones específicas en la estructura social capitalista de los involucrados en las relaciones de poder que construyen tecnologías y, asimismo, de aspectos culturales y representacionales que afectan la adopción y construcción de unas tecnologías en lugar de otras. Se eliminan, gracias a esto, las representaciones de los grupos como iguales, esto es, la indiferenciación entre grupos sociales que participan en el diseño y construcción de las tecnologías.

Adicionalmente, los grupos sociales relevantes ayudan a pensar en un grupo omitido o ignorado en las proposiciones constructivistas, el que he propuesto llamar "grupos sociales heterónomos".9 Estos son, como hemos visto, aquellos que no participan directamente, o en forma consciente, en el diseño y construcción de tecnologías. Estos grupos también tienen representaciones clasistas específicas; en este respecto, cabe aclarar que el aspecto clasista no excluye a capitalistas de ser parte de estos grupos, aunque, en cierta forma, si tiende a imponerse a las clases trabajadoras. ${ }^{10}$ Además, la pertenencia de clase limita fuertemente las estrategias políticas a realizar en caso de querer dejar su carácter heterónomo. En este sentido, el aspecto de clase tendría que ser considerado en cada investigación particular.

9 "Grupos sociales irrelevantes" los ha llamado Winner (1993, p. 369-70). Ante las críticas, Bijker (1995, p. 48) mencionó su existencia denominándolos "grupos sociales sin poder", aunque este último concepto fue propuesto para dar cuenta de aquellos cuyas voces no podían ser escuchadas en la "flexibilidad interpretativa". Yo he decidido proponer este concepto para señalar dos aspectos: uno tiene que ver con que, a pesar de esta mención por parte de Bijker, la existencia de estos grupos es casi inexistente en la literatura constructivista y, por otro lado - y más importante -, hacer mención a las reglas y leyes presentes en las tecnologías (representado por el sufijo “-nomos") y, junto con ello, que esas reglas y leyes son exteriores a esos sujetos ("hetero-"), sometiéndose con ello a la incorporación de valores propios de otros grupos o clases sociales o fracciones de clase. Valores estrechamente ligados con sus necesidades de mantener su posición hegemónica en la sociedad, o bien, con los requerimientos del capital. 10 En el caso de las tecnologías digitales libres o abiertas, dadas sus condiciones específicas, esta imposición a las clases trabajadoras es profundamente limitada. 
Los grupos heterónomos incluyen a la inmensa mayoría de la sociedad. Esto se evidencia al tener en cuenta los procesos de construcción de tecnologías, donde, usualmente, es muy limitada la cantidad de grupos participando activamente. Es justamente porque son ellos los que representan a la gran mayoría en la sociedad que las tecnologías poseen apariencia de autonomía y, en definitiva, una existencia cuasi-concreta como autónomas.

De esto último se sigue otro de los aspectos que he propuesto suspender de la perspectiva constructivista: la afirmación acerca de la imposibilidad de establecer limites entre tecnología y sociedad. Una de las consecuencias fundamentales de poder emprender esta distinción se observa en la posibilidad de diferenciar entre grupos que se ven heteronomizados por las tecnologías que gobiernan sus vidas. Como este elemento ya lo he tratado con detalle más arriba, aquí sólo lo menciono para tenerlo presente como uno de los puntos a modificar en favor de una propuesta teórica comprometida políticamente.

Puede considerarse, adicionalmente y al respecto de los grupos heterónomos, otra consecuencia de su consideración e inclusión en los análisis. Siendo que se caracterizan por asumir e incorporar los valores, reglas y leyes (nomos) desarrollados por otros, y, además, que su participación se produce a través de vías implícitas, conviene recordar ese aspecto para exponer consecuencias teóricas al respecto. He mencionado que algo central en la crítica de las tecnologías se encuentra en la identificación, por un lado, del contenido popular de las tecnologías construidas o en construcción y, por el otro, (utilizando el término de Zizek) de la distorsión ideológica que permite la conservación de las relaciones de poder establecidas. Con esto he querido decir que, en términos concretos, la creación de tecnologías implica generalmente la imposición de valores específicos que ayudan al sostenimiento del orden social imperante, pero cubiertos de un manto de valores sensiblemente importantes para los usuarios. Para concretizar aún más esta idea, es interesante analizar los modos de incorporación de esos valores a las tecnologías a través de una revisión del concepto de "código técnico", propuesto por Feenberg.

Para Feenberg, el código técnico implica la "realización de un interés bajo la forma de una solución técnicamente coherente a un problema” (Feenberg, 2005, p. 114). Representa la articulación entre necesidades sociales y técnicas, gracias a la inclusión de valores técnicos a las tecnologías y a la aplicación de eficiencia técnica y racionalidad técnica. En otras palabras, a través del principio de racionalidad técnica se introducen elementos de interés particular (valores) de los grupos en cuestión a las tecnologías. El código técnico da cuenta de esos valores e intereses propios de un grupo o varios grupos sociales cristalizados en un artefacto técnico. Para Feenberg, cuando estos códigos técnicos 
están reforzados por la percepción que los individuos tienen acerca de su propio interés y de la ley, su significado político generalmente pasa desapercibido. Eso es lo que significa decir que un cierto modo de vida está culturalmente asegurado y que el poder correspondiente es hegemónico. Así como la filosofía política cuestiona las formaciones culturales que se han arraigado a sí mismas en la ley, la filosofía de la tecnología cuestiona las formaciones que se han arraigado a sí mismas en códigos técnicos (Feenberg, 2005, p. 114).

Por lo tanto, la identificación de valores no es otra cosa que la identificación de códigos técnicos. La cuestión central será identificar esos valores incorporados y analizarlos críticamente. El contenido auténtico y la distorsión ideológica de la que habla Zizek, de este modo, tienen su correlato en la filosofía de la tecnología en el concepto de código técnico. La crítica, sostengo, debe encaminarse a poder deconstruir los valores incorporados a la tecnología de manera tal que sea posible distinguir los elementos que pretenden contribuir al sostenimiento de la explotación y de la dominación hegemónica.

Por su parte, el concepto de "marco tecnológico" es, a mi entender, de fácil conservación, siempre y cuando se lo alinee con lo antedicho. De ese modo, un marco tecnológico puede ser visto como el paradigma o valores generales creados en torno a una serie de objetos técnicos específicos. Ante la consideración del capitalismo y del mercado, el marco tecnológico se encontraría en un nivel intermedio, sirviendo de contexto cultural-técnico para el desarrollo de un conjunto de tecnologías específicas. En este esquema, el concepto sufriría una conversión al punto de representar un conjunto de valores presentes en el ambiente de desarrollo de ciertas tecnologías específicas, reproduciendo, por ejemplo, algunos aspectos de la lógica mercantil o, en algunos casos menores, contradiciéndola. De este modo, las tecnologías digitales libres, por ejemplo, estarían impregnadas por su propio marco tecnológico donde los valores fundamentales se encuentran en la defensa de la apertura de la tecnología, la liberación de la información y de nuevos desarrollos, el trabajo colaborativo y la solidaridad. Esos valores, por lo tanto, representan códigos técnicos específicos. En consecuencia, se observa que ambos conceptos (código técnico y marco tecnológico) poseen una estrecha relación: los marcos tecnológicos orientan conductas en la construcción de tecnologías y los códigos técnicos, por su parte, representan esos valores ya incorporados o cristalizados en las tecnologías.

Finalmente, cabe mencionar un elemento no rechazado del enfoque constructivista analizado. El elemento más problemático y que, al menos en posiciones en favor del compromiso político no cabe sostener, es el relativismo. Ya he mencionado los que, a mi entender, son los problemas del relativismo, aquí sólo me interesa mencio- 
nar que, al contrario de lo que sucede con otros conceptos del herramental teórico del enfoque Scot, el relativismo no puede ser conservado bajo ningún concepto. No existen modificaciones mínimas ni profundas que permitan su reutilización en enfoques comprometidos políticamente y, por lo tanto, es un elemento que atenta en contra del giro en favor del compromiso mencionado.

\section{Conclusión}

El presente texto ha intentado ser una exposición de los elementos a conservar de la tradición constructivista y, particularmente, del enfoque Scot, para poder alimentar el llamado giro normativo o giro participativo que he preferido llamar, siguiendo a Söderberg (2010), giro en favor del compromiso político en los estudios de la tecnología. La elección del enfoque Scot tiene que ver, básicamente, con su potencial para poder alimentar este giro siempre y cuando se realicen una serie de modificaciones de base. Esas modificaciones son las que aquí he expuesto.

Asimismo, en el presente texto, se ha podido visibilizar la tensión entre dos modos posibles de ver el objeto de estudio: por un lado, el constructivista, con una tradición filosófica que se asienta en el relativismo y en la duda frente a conceptos generales o totalizantes. Por otro lado, el alineado con la teoría crítica, asentada en Marx y Hegel, y a favor de conceptos generales y totalizantes. Ante este esquema, no han habido dudas en seleccionar la segunda de las vías. Varios autores se han alineado en esta nueva senda, algo que resultaba más difícil de hacer hace dos décadas. Aquí me he centrado fundamentalmente en dos de ellos: Andrew Feenberg y Johan Söderberg. La vuelta del marxismo, un nuevo nacimiento luego de tantas muertes, torna posible y habilita esta elección. Después de todo, el capitalismo no ha muerto y la última muerte de Marx ha sido muestra expresa de la buena vida del sistema o, más bien, de la inexistencia de oposiciones fuertes a la lógica del capital durante los años de expansión del llamado neoliberalismo.

En esta línea, la defensa de los conceptos de clase (reafirmado, como he señalado, por Feenberg) de mercado y de su consecuente relación con la dinámica del intercambio de mercancías (Söderberg), además de la defensa del concepto de lógica del capital, obedece a la necesidad, según entiendo, de alimentar al giro mencionado con la tradición filosófica de la teoría crítica de la tecnología.

La inclusión de esos elementos ha guiado el rechazo absoluto de algunos conceptos de la perspectiva Scot o, en otros casos, las modificaciones conceptuales indicadas. Así, el concepto de grupo social relevante ha sido planteado como de fácil con- 
servación, siempre y cuando se lo relacione con cuestiones estructurales de fondo que posibiliten concebir las diferenciaciones que entre ellos, y en su interior, existen. En este sentido, se ofreció conservarlo mediante su vinculación a los conceptos de clase (y, alternativamente, de gerenciadores y gerenciados), lo que permitiría observar las relaciones de poder entre los que construyen efectivamente y los que ordenan la construcción. Además, el concepto de clase se lo tomó en un doble sentido, como inclusivo de las propias identificaciones de los sujetos y, por otro lado, como expresión de las posiciones particulares de clase en la estructura capitalista. Así, los grupos sociales relevantes deben estudiarse pero considerando su identificación con las fracciones de clases específicas.

Sin querer realizar un resumen del presente texto, me limito ahora a realizar las últimas conclusiones sobre la importancia de criticar la autonomía ficticia o ilusoria de las tecnologías, algo omitido en el enfoque Scot. Esta omisión se produce, según he señalado, como consecuencia, en primer lugar, de los problemas acarreados por la indiferenciación teórica entre tecnología y sociedad, en segundo término, como consecuencia del rechazo absoluto de categorías (incluso mínimamente) determinantes, y, en consecuencia, a causa de la no consideración de criterios estructurales. Esto significa que esta omisión es totalmente consecuente con las bases teóricas generales del enfoque. En este sentido, esta afirmación supone otro elemento de peso para poder establecer la necesidad de criticar las bases teóricas relativistas y particularistas del constructivismo. Si lo que se busca es criticar las tecnologías como dadas y la generación de sujetos heterónomos, es necesario abandonar esos principios, abogando por la preeminencia de criterios estructurales.

En este sentido, la apuesta en favor de la aplicación de conceptos estructurales es otra cuestión a destacar. En el artículo he propuesto la incorporación de estos elementos en los estudios sobre tecnología. La estructura, bajo ningún concepto, implica que las acciones estén predefinidas o predeterminadas, es decir, que sólo unas acciones son posibles. Por el contrario, la contingencia aún juega un rol esencial y fundamental en el desarrollo tecnológico. La cuestión se encuentra en que la contingencia no es absoluta, no es total. Si para la totalidad tenemos al capital y a su lógica de funcionamiento (lo que implica a las relaciones de intercambio mercantil), entonces la contingencia se limitará a las posibilidades brindadas por esa totalidad. Con esto he intentado destacar los factores particulares que sólo pueden ser apreciados a través de la investigación de campo, mediante la indagación de los particulares. De todos modos, y consecuentemente, esos factores particulares tendrán que ser remitidos a situaciones contextuales más amplias, más amplias aún que el contexto dado por los marcos tecnológicos, esto es, el capitalismo.↔ 


\title{
Horacio Correa Lugero
}

Centro de Investigaciones sobre Economía y

Sociedad en la Argentina Contemporánea, Universidad Nacional de Quilmes, Argentina. hecorrealucero@gmail.com

\section{Studies on science, technology and society: in favor of political commitment}

\begin{abstract}
ABSTRAGT
The paper mentions the turn in favor of participatory or politically engaged approaches in the STS field, and outlines a proposal for deepening this turn within a critical theory of technology route. Inspired in the contributions of Andrew Feenberg and Johan Söderberg, the paper undertakes this task through a combination of concepts from the perspective of social construction of technology with those of a Hegelian-Marxist tradition interested in visions of the capitalist system as a whole posing limits to contingency in technological development. Thus, it offers to rescue such concepts as class, capitalism, market relations and logic of the capital, to better understand technological development. During this task, constructivist relativism is considered a central problem to deepen this turn and thus replaced by an assessment of the technical codes and the wider social context represented by capitalism. In the following parts the paper presents a reuse of constructivist concepts according to the interests of a position aligned with critical theory of technology. Thus, the concept of "technological frame" will be associated with Feenberg's "technical code" and with the rescue of the market logic advocated by critical theory and Söderberg.
\end{abstract}

KEYWords $\bullet$ Participatory turn. Politically engaged turn. Critical theory of technology. Scot. Technological frame. Technical code. Market logic. Logic of the capital. Capitalism.

\section{REFERENGIAS BIBLIOGRÁFICAS}

Balibar, E. La crainte des masses: politique et philosophie avant et après Marx. Paris: Galilée, 1997.

Bijker, W. E. Do not despair: there is life after constructivism. Science, Technology \& Human Values, 18, 1 , p. $113-38,1993$. . Ofbicycles, bakelites, and bulbs: Toward a theory of socio-technical change. Cambridge: The MIT Press, 1995 .

. The need for public intellectuals: A space for STS. Pre-Presidential Address, Annual Meeting 2001. Science, Technology, \& Human Values, 28, 4, p. 443-50, 2003.

Bijker, W. E.; Hughes, T. P. \& Pinch, T. J. (Ed.). The social construction of technological systems. New directions in the sociology and history of technology. Cambridge: The MIT Press, 1987.

BLoor, D. Wittgenstein and Mannheim on the sociology of mathematics. Studies in History and Philosophy of Science, 4, 2, p. 173-91, $197^{3}$. 
Bloor, D. Knowledge and social imagery. Chicago: University of Chicago Press, 1991.

Castoriadis, G. Transformación social y creación cultural. El Psicoanalítico, 6, p. 155-75, 2012.

Feenberg, A. Questioning technology. New York: Routledge, 1999.

Teoría crítica de la tecnología. Revista Iberoamericana de Ciencia, Tecnología y Sociedad, 2, 5, p. 10923, 2005.

FulLER, S. Philosophy, rhetoric, and the end of knowledge: the coming of science and technology studies. Madison: University of Wisconsin Press, 1993.

Grüner, E. El retorno de la teoría crítica de la cultura: una introducción alegórica a Jameson y Zizek. In: Jameson, F. \& Zizex, S. (Ed.). Estudios culturales. Reflexiones sobre el multiculturalismo. Buenos Aires: Paidós, 2008. p. 11-64.

Hacketт, E. J. et al. (Ed.). The handbook of science and technology studies. 3 ed. Cambridge: The MIT Press, 2008.

Hughes, T. The evolution of large technological systems. In: Bijker, W. E.; Hughes, T. P. \& Pinch, T. J. (Ed.). The social constrution of technological systems. New directions in the sociology and history of technology. Cambridge: The MIT Press, 1987. p. 51-82.

La evolución de los grandes sistemas tecnológicos. In: Thомаs, H. \& Buch, A. (Ed.). Actos, actores y artefactos. Buenos Aires: Universidad Nacional de Quilmes Editorial, 2008. p. 101-45.

Jameson, F. \& Zizex, S. (Ed.). Estudios culturales. Reflexiones sobre el multiculturalismo. Buenos Aires: Paidós, 2008.

JASANOFF, S. Technologies of humility: citizen participation in governing science. Minerva, 41, 3, p. 223$44,2003$.

KLein, H. K. \& Kleinman, D. K. The social construction of technology: structural considerations. Science, Technology \& Human Values, 27, 1, p. 28-52, 2002.

Latour, B. Why has critique run out of steam? From matters of fact to matters of concern. Critical Inquiry, 3o, 2, p. 225-4, 2004.

LAw, J. The Greer-Bush test: on politics in STS. Draft paper, version of 23rd December, 2009. Disponible en: $<$ http://ww.heterogeneities.net/publications/Law2009TheGreer-Bush Test.pdf $>$. Acceso en: 24 sep. 2014 .

Lynch, M. \& Cole, S. Science and technology studies on trial: dilemmas of expertise. Social Studies of Science, 35 , 2, p. 269-311, 2005.

Pinch, T. J. \& BijKer, W. E. The social construction of facts and artifacts: or how the sociology of science and the sociology of technology might benefit each other. Social Studies of Science, 14, 3, p. 399-441, 1984 .

Marx, K. El 18 brumario de Luis Bonaparte. Madrid: Fundación Federico Engels, 2003.

Rosen, P. The social construction of mountain bikes: technology and post-modernity in the cycle industry. Social Studies of Science, 23, 3, p. 479-513, 1993.

Sismondo, S. Science and technology studies and an engaged program. In: HAckett, E. J. et al. (Ed.). The handbook of science and technology studies. 3 ed. Cambridge: The MIT Press, 2008. p. 13-32.

SöDERBERg, J. Reconstructivism versus critical theory of technology: alternative perspectives on activism and institutional entrepreneurship in the Czech wireless community. Social Epistemology, 24, 4, p. 239-62, 2010.

Tномаs, H. Estructuras cerradas versus procesos dinámicos: trayectorias y estilos de innovación y cambio tecnológico. In: Tномаs, H. \& Buch, A. (Ed.).Actos, actores y artefactos. Buenos Aires: Universidad Nacional de Quilmes Editorial, 2008. p. 217-62. 
Tномаs, H. De las tecnologías apropiadas a las tecnologías sociales. Conceptos/estrategias/diseños/ acciones. Ponencia presentada en la Primera Jornada sobre Tecnologías Sociales, Programa Consejo de la Demanda de Actores Sociales, Ministerio de Ciencia, Tecnología e Innovación Productiva. Buenos Aires, 2009. Disponible en: <http://inti.gob.ar/bicentenario/documentoslibro/pdf/anexo_4/jornadas_tecno_soc_hernan_thomas.pdf $>$. Acceso en: 24 sep. 2014.

Thомаs, H. \& Buch, A. (Ed.). Actos, actores y artefactos. Buenos Aires: Universidad Nacional de Quilmes Editorial, 2008.

Thomas, H.; Fressoli, M. \& Lalouf, A. Presentación. Estudios sociales de la tecnología: ¿hay vida después del constructivismo? Redes, 14, 27, p. 59-76, 2008.

Winner, L. Upon opening the black box and finding it empty: social constructivism and the philosophy of technology. Science, Technology \& Human Values, 18, 3, p. 362-78, 1993.

Zızeк, S. Multiculturalism, or, the cultural logic of multinational capitalism. New Left Review, 225, p. 28$5^{1,1997 .}$

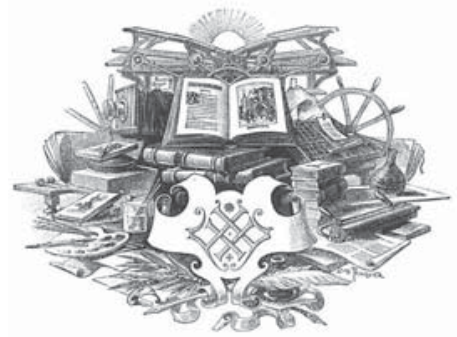

